Epidemiological Monitoring of AIDS, ${ }^{1}$ may more appropriately describe HIV-infected patients requiring hospitalisation.

V SORIANO E VALENCIA J GONZÁLEZ-LAHOZ Service of Infectious Diseases, Instituto de Salud Carlos III, Madrid
Spain

Address correspondence to: Dr Vincent Soriano, C/ Rafael Calvo $7,2^{\circ}$ A, 28010-Madrid, Spain.

1 Ancelle-Park R. Expanded European AIDS case definition. Lancet 1993;341:441.

Accepted for publication 14 April 1994.

Chlamydia trachomatis infection in males attending general practitioners

In males, $C$ trachomatis is the most common cause of non-gonococcal urethritis and cases of epididymitis and proctitis. ${ }^{1}$ Until recently tissue culture isolation from urethral swabs was the primary laboratory procedure for detection of $C$ trachomatis in males. Several non-culture methods of detecting the organism, mainly based upon immunological techniques, have replaced tissue culture in many diagnostic laboratories. The two most common techniques for detecting chlamydial antigen are direct immunofluorescence (DIF) and enzyme immuno-assay (EIA). Examination of first void urine (FVU) samples using EIA to detect $C$ trachomatis antigen appears to be as sensitive as taking urethral swabs for diagnosis in men. ${ }^{2}$

We have studied chlamydia prevalence in males attending their general practitioners from whom a mid stream urine sample (MSU) was submitted for routine bacteriology for suspected urinary tract infection. Direct microscopy and culture were first performed. Samples which had more than 5 pus cells per high power field but no bacterial growth were tested for chlamydia antigen in an EIA (Dako) and all repeatedly reactive samples were confirmed by DIF using fluorescein labelled monoclonal antibody (Syva, Microtrak). The DIF was read by two observers. A preparation showing at least two or more chlamydial elementary bodies (EB) was defined as positive.

A total of 293 patients were studied over a period of 18 months. Eighteen samples were positive for $C$ trachomatis (table). A further four samples were reactive in the EIA but could not be confirmed by DIF and were

Age distribution of patients with $C$ trachomatis infection

\begin{tabular}{lll}
\hline Age in years & No. of patients tested & No. positive (\%) \\
\hline All & 293 & $18(6)$ \\
$\leqslant 30$ & 99 & $15(15)$ \\
$\leqslant 40$ & 158 & $17(11)$ \\
$>40$ & 135 & $1(0.7)$ \\
\hline
\end{tabular}

therefore considered as negative for the purpose of this study.

Overall, $6 \%$ patients with sterile pyuria were positive for $C$ trachomatis. However, $15 / 18$ (83\%) were from males under 30 years; $15 \%$ of all urines submitted from that age group were positive for chlamydia. In an earlier report ${ }^{3} 19 \%$ of patients with sterile pyuria were reported as chlamydia positive but there was no information on type or age distribution of the patient population. It is possible that dysuria and pyuria in the patients we studied was an indication of urethritis rather than UTI. Chlamydial infection was not suspected in these patients as urethral swabs were not submitted. Asymptomatic or unsuspected chlamydial infection is an important link in spread of infection. Many of these patients may not perceive themselves at risk or may be reluctant to attend genitourinary clinics. Although urethral swabbing is considered by many as invasive, a urine sample is a noninvasive sample and simplifies the investigations for $C$ trachomatis. In our opinion routine testing for $C$ trachomatis of urine samples with sterile pyuria from males, especially under 30 years of age, is of clinical benefit. The relationship between patients' presenting symptoms and chlamydia infection needs to be investigated further. Many general practitioners already screen women for chlamydia infection, and the comparative sensitivity of urine samples makes the diagnosis of chlamydial infection in males easily accessible.

$$
\begin{array}{r}
\text { G KUDESIA } \\
\text { P M ZADIK } \\
\text { M RIPLEY } \\
\text { Regional Public Health Laboratory, } \\
\text { Herries Road, Sheffield S5 } 7 B Q \text { UK }
\end{array}
$$

Address correspondence to: Dr G Kudesia

1 Taylor-Robinson D, Munday PE. Chlamydial infections. In: Oriel JD, Waugh $M$ (ed) Anglo-Scandinavian Conference on Sexually transmitted diseases. London: Royal Society of Medicine Services Ltd, 1988:3-12.

2 Chernesky M, Castriciano S, Sellors J, et al. Detection of Chlamydia trachomatis antigens in urine as an alternative

3 Matthews RS, Wise R. Non-invasive sampling method for detecting Chlamydia trachomatis. Lancet 1989;i:96.

Accepted for publication 21 March 1994.

\section{Genital ulceration secondary to Epstein Barr virus infection}

We wish to report the case of a 13 year old girl who presented to the Christchurch Sexual Health Centre in January 1994 with marked genital ulceration secondary to an acute Epstein Barr virus (EBV) infection. She had been previously well and was on no current medication. She was not sexually active and denied oral-genital contact from a stable boyfriend who himself was asymptomatic.

She presented with fever and dysuria and examination of the vulva revealed three very large deep genital ulcers on the labia minora which had vivid purple borders. Shotty inguinal lymph nodes were present and her 